\title{
VIABILIDAD DE UNA PROPUESTA DE CAMBIO PARA EL MEJORAMIENTO CONTINUO EN UNA ORGANIZACIÓN COMPLEJA: DEPARTAMENTO DE ENFERMERÍA
}

\author{
Nora Agnes Veja Villalobos* \\ Alacoque Lorenzini Erdmann**
}

VILLALOBOS, N.A.V.; ERDMANN, A.L. Viabilidad de una propuesta de cambio para el mejoramiento continuo en una organización compleja: Departamento de Enfermería. Rev.latino-am.enfermagem, Ribeirão Preto, v. 4, n. 3, p. 89-98, dezembro 1996.

El departamento del Enfermería es una organización compleja, que tiene su cultura, y que se compone de dos o más grupos enlazados por una red compleja de relaciones sociales, valores y metas comunes y sirven como contexto de las intervenciones de enfermería. La organización compleja envuelve tipo, subsistema, fronteras, filosofía, y estilo de vida, proceso de adaptación al entorno, integración, toma de decisiones, tratamiento de la información, comunicación, coalición-formación y compromiso. La propuesta teórica de cambio para el mejoramiento contínuo está basada en los siete pasos de la teoría de cambio de Lippitt y en el P.C.D.A.

TÉRMINOS CLAVES: organización compleja, estructura, proceso, cambio, mejoramiento continuo.

\section{INTRODUCCIÓN}

El departamento de enfermería tiene en su que hacer algo de la teoría clásica: es normativa y prescriptiva; como hacer bien las cosas; impregnada de principios y recetas aplicables a todas las circunstancias, llevando en cuenta una apreciable dosis de buen sentido.

\footnotetext{
* Profesora Asociada, Escuela de Enfermería, Universidad de Costa Rica y post graduanda en enfermería, UFSC.

** Profesora Titular, Universidad Federal de Santa Catarina
} 
La administración del Departamento se ha visto empujada por un enfoque más contingencial, aunque no es al que se aplica. La teoría Contingencial da énfasis al ambiente y a las demandas ambientales sobre la dinámica organizacional.

La filosofía del mejoramiento continuo es una nueva forma de administrar una organización, implica mudanza de cultura que solo podrá ocurrir por un liderazgo altamente comprometido que se comporte de manera que su teoría sea comprobada en la práctica, creando un ambiente donde las energías creativas de todos los funcionarios puedan ser mejor utilizadas.

Un proceso de cambio en un primer momento provoca una cierta angustia porque lo viejo ya no es y lo nuevo no está claro. Las reglas antiguas no deben continuar y las nuevas se están construyendo. La alta administración debe asumir que es hora de mudar, pero para decidir qué y cómo hacerlo debe hacerse un diagnóstico organizacional, que determine el ritmo, la amplitud y las prioridades en la operacionalización del mejoramiento continuo.

Al elaborar un programa de mejoramiento continuo es importante respetar la cultura de la casa (Departamento de Enfermería), lo que valoriza, lo que privilegia, cuáles son los principios éticos que están más absorbidos, cómo es el reracionamiento entre las personas. Cultura es un proceso dinámico que permanentemente está en evaluación, lo que necesitamos reforzar o redireccionar porque está en el camino correcto (WERNECK, 1994, P.73).

El Departamento de enfermería tiene su cultura, es una organización compleja. La organización compleja es un tipo de sistema social que se compone de dos o más grupos enlazados por una red compleja de relaciones sociales, valores y metas comunes. Sirven como contexto de las intervenciones de enfermería centradas en el individuo, la familia y el grupo.

Por ello proporcionan el entorno en el que las enfermeras y los clientes van todos a promover y restablecer la salud, prevenir la enfermedad y la invalidez y a encargase del aprendizaje relacionado con la conducta sana.

La enfermería es una integración de muchos procesos en un sistema complejo en el que participan en igual medida la enfermera y el cliente.

La síntesis de: 1) Las destrezas básicas del proceso: Percepción, comunicación, cuidado, conocimiento, solución de problemas, normatización, creatividad, evaluación; 2) Los componentes de la práctica: valoración, intervención y evaluación; y 3) Las destrezas del cambio: "armamentarium" principal de la enfermera que funciona como agente de cambio con individuos, familias, grupos, organizaciones complejas y comunidades; representa un enfoque de proceso para la práctica de la enfermería.

En la teoría general de sistemas, un sistema se define "como un conjunto de elementos o unidades interactuando entre sí dentro de una frontera que filtra la clase y el régimen de circulación de entradas y salidas hacia el sistema y desde él." (HALL \& REDDING, 1990, P.24)

Esta definición estipula que los sistemas poseen estructura y función o proceso. 
La estructura de un sistema es la ordenación estática de las partes de un sistema en cualquier momento en el espacio tridimensional. La estructura de un sistema social se refiere a esos elementos que dan al sistema sus características que persisten a lo largo del tiempo, como: tipo, subsistemas, fronteras, filosofía, estilo de vida.

El proceso es el cambio dinámico en la materia, la energía o la información de ese sistema en el tiempo. Los procesos son los conjuntos de cambios colocados en un orden especial que ocurren continuamente en el tiempo.

El proceso para identificar las fuerzas, los problemas y las intervenciones necesarias para facilitar el funcionamiento organizacional.

El proceso tiene que ve con: Adaptación al entorno, la integración de los subsistemas, la toma de decisiones, el procesamiento de la información, la comunicación, coalición-formación, compromiso.

Analizaremos el sistema en una organización compleja como es el Departamento de Enfermería según estructura y proceso.

En cuanto estructura considerase:

- Tipo: El departamento de enfermería tiene sus propios estatutos y reglas que provienen del supra sistema cujo presupuesto es estatal. Es una organización legal-burocrática con un uso racional de autoridad; clara identificación y distribución de poder; establecimiento de fronteras geográficas, legales y funcionales; políticas bien definidas y procedimientos que dirigen las operaciones día a día; gran interés por la jerarquía y seguimiento de la cadena de comandos en cuanto a responsabilidad, autoridad, status y recompensas; división especializada de trabajo y sentido de continuidad a partir de la estabilidad de los roles e posiciones dentro de la organización. La práctica de enfermería está dada por servicio para pacientes ingresados en el hospital con manejo ambulatorio de la prevención terciaria. Es un hospital especializado no lucrativo. El propósito establecido es brindar atención especializada según las causas de morbimortalidad; Perinatología, malformaciones congénitas, tumores, cirugías, infecciones. Relación entre propósito, filosofía, política, procedimientos, prácticas y producto son coherentes.

- Subsistema: el departamento de enfermaría tiene su propio organigrama (estructura formal). Es un departamento independiente con relación jerárquica hacia el director del Hospital. En estructura informal el poder de la organización que transacciona el quehacer está dado por un sistema de supervisión coercitiva, poco operacional que mide cantidad de atención.

- Fronteras externas: Hay apertura relativa, renuencia al cambio, al trabajo con otros miembros del equipo. Generalmente la relación es de rigidez, destinada la enfermera solo al cuidado del paciente. Excepto las que están en programas especiales.

- Fronteras internas: Se acepta a enfermeras diplomadas, bachilleres, licenciadas, auxiliares tituladas, niñeros entrenados, pero no se acepta cambios propuestos por el personal para mejorar la atención del paciente hospitalizado depende de las demandas médicas. 
- Filosofía: el departamento de enfermería cuenta con filosofía escrita pero en la práctica se ha observado que han empezado a operar primero los intereses de los trabajadores sobre el interés principal que es el cuidado óptimo del niño. Como los padres de familia participan en el cuidado, solo se les realiza lo de "enfermería", la relación es de recordatorio sobre lo único que hace enfermería, es punitivo hacia las exigencias de los padres de familia. La enfermera jefe es la encargada pero en realidad no tiene relación directa con el cliente. Quien lo hace es el personal menos calificado porque son los auxiliares de enfermería con el médico quienes tienen la responsabilidad del cuidado directo del cliente y las acciones para lograr su progreso. Las enfermeras sienten que son explotadas pero porque solo administran medicamentos y se encargan de anotar indicaciones médicas y trámites administrativos a pesar de que cuenta cada unidad con una secretaria.

- Estilo de vida: En la asistencia de enfermería es la enfermera jefe de cada unidad la responsable de la calidad del cuidado del paciente. Hay una descripción del trabajo para cada puesto pero de acuerdo a la unidad lo que impera es lo que cada equipo considera que debe ejecutar cada miembro. En neonatología por ej. la que se dice que "administra"se encarga de las coordinaciones, del Kardex y de administrar medicamentos. Las otras dan cuidado directo junto con el resto de personal de la unidad. Pero en las otras unidades de medicina y cirugía, el cuidado directo está en su mayoría en manos del personal auxiliar y la enfermera solo administra medicamentos y anota órdenes médicas. No hace anotaciones en la historia clínica. La supervisión sobre del personal auxiliar es sobre el cumplimiento o no de órdenes médicas y no en la valoración de la calidad del cuidado brindado. Se pelea por ocupar el rol pero sobre la base del título que se posee y no por la diferencia en la atención.

En el proceso:

- Adaptación al entorno: El departamento de enfermería emplea el personal necesario para la asistencia. Sin embargo ocurren dos casos: a) El enfermero no gusta de empiearse por el ambiente que se vive dentro del sistema de enfermería del hospital. b) Las plazas que se ofrecen dentro del presupuesto no llenan las expectativas de acuerdo a la cantidad y calidad de clientes que son atendidos en cada unidad. Se refrena la asistencia a clientes con mejores condiciones pero no hay una adecuada educación que permita el egreso hospitalario satisfactorio para el cliente que prevenga un futuro ingreso. Se refrena por cantidad pero no con programas de reorientación que permitan estimular en el cliente un nivel óptimo de salud. El departamento no tiene metas claras para retener al personal bien calificado con preparación y capacidad para ocupar el papel que le corresponde en la asistencia del cliente y en el establecimiento de políticas de atención. El departamento de enfermería debe distribuir al personal de enfermería según sus habilidades, sin embargo lo hace por cantidad de pacientes según unidades. Se distribuye según asistencia al trabajo sin un sistema de motivación y estímulo por años de servicio, por responsabilidad.

- Integración: El departamento de enfermería interviene en la atención directa del 
paciente pero no puede influir en los otros departamentos para que la coordinación de actividades sea pronta y efectiva. No puede tampoco tener una política para el ingreso de pacientes a cirugía por ejemplo con un sistema bien planificado para la atención oportuna. Los logros para la coordinación con otros departamentos está dado por la amistad y el trueque por intereses particulares que benefician a las personas más cercanos que tienen influencia. Cuando hay conflicto con un departamento casi siempre el perjudicado es el paciente porque auenque precise se deja a lo último para su resolución.

- Toma de decisiones: El departamento de enfermería no es el que toma la decisión directa en la asistencia de enfermería. La calidad, rapidez, precisión de la asistencia está dada por el actuar del médico responsable de los pacientes de una unidad. Es él el que fija que es lo que se quiere en la asistencia. La asistencia de enfermería está dada por el dejar hacer o buscar responsables por lo que no se desarrolla bien. No se tiene una clara visión de la necesidad de tomar decisiones oportunas para el cliente y su familia, que disminuya además los costos de operación. Esto provoca en el departamento de enfermería un entrebamiento de la acción supeditada a los intereses de otros grupos.

- Tratamiento de la información: La información de la asistencia de enfermería no está estructurada formalmente. No se cuenta con un sistema de registro de los aciertos y desaciertos en la atención; ni se maneja los índices de morbimortalidad, los días de estancia. Las acciones a tomar por enfermería están en función de los datos de otros departamentos y de los caprichos de otros. El personal de cada unidad no maneja la inmensidad del océano en el que navega. No se realiza previsiones del sistema de información de beneficio para enfermería ni los sistemas de computarización que posee la institución.

- Comunicación: Generalmente la única efectiva dentro del departamento es la que se refire al horario de trabajo, a los libres y a los cambios de turno. Las relaciones de discusión en reuniones es para manifestar el descontento o tomar la actitud de indiferencia. El personal está a la defensiva a la hora de distribuir es trabajo, de ejecútalo y casi es nula la comunicación en la evaluación de la asistencia proporcionada.

- Coalición-formación: El poder adscrito al departamento de enfermería es el que está determinado al ser el responsable del cuidado continuo y directo de los pacientes internados y los ambulatorios. El vigilante que tiene la obligación de responder por lo que está sucediendo. El poder logrado prácticamente no existe porque el enfermero se supedita a las órdenes médicas para el desarrollo de sus actividades. Excepto casos espontáneos no todos los profesionales se preocupan por tener un poder logrado, ya que implica responsabilidades que no están dispuestos a asumir. Deben demostrar la competencia de su status profesional dada en su formación, desarrollada en su trabajo y que no aflora casi nunca. La coalición con otros profesionales es casi nula puesto que solo se reduce a la comunicación de órdenes médicas pero no al compartir logros en la atención prestada. Además para obtener el poder logrado se requiere de esfuerzo constante para mantener e 
incrementar el conocimiento y las habilidades. Esto algo que en el departamento de enfermería ha salido de los objetivos propuestos para el grupo. En el juego social del poder generalmente es enfermería la que termina siendo controlada por las otras subordinadas, acceden a sus deseos y los apoyan. Pocas son las que manejan relaciones públicas independientes y con control sobre los demás. El juego de status es lo que enfermería desarrolla a través de la publicidad del título universitario que se posee. Ha surgido una corriente de obtención de títulos por competencia de poder, status dinero pero no por desarrollo del conocimiento y de la pericia profesional. El juego del dinero no se da abiertamente puesto que la institución no tiene clientes privados. Y los que hay por la unidad de "pensiones" provocan un rechazo en la mayoría por las demandas de atención. El juego intelectual es algo que enfermería no le da importancia porque no obtiene ganancia de poder o cambio. Prácticamente no lo ha experimentado porque provoca stress y un esfuerzo adicional a las 8 horas de atención directa. Implica trabajar en investigación, en congresos, seminarios y es algo que le demanda una dedicación que no está dentro de sus expectativas de vida. El juego sexual, el hostigamiento es algo que se da de una forma encubierta y también pública. Todo el mundo sabe que así se manejan las coordinaciones, los trueques pero nadie lo admite claramente.

- Compromiso: Es algo que en el ambiente no se respira puramente. El interés mutuo por el mejoramiento de la asistencia de enfermería, del departamento no se da, solo se sobrevive en el sistema. Entre corrillos se escucha la insatisfacción en el empleo, por lo que se ejecuta. El conflicto se maneja in apropiadamente con sanciones pero sin esclarecimiento de las razones que la ocasionaron. Hay alto índice de rotación de personal, ausentismo, mal servicio a los clientes, calidad mínima de cuidado. Las personas problema son premiadas al transferirlas a unidades de menos riesgo, mejor horario.

\section{OBJETIVO}

Establecer una propuesta teórica de cambio, para el mejoramiento continuo de una organización compleja como lo es el Departamento de Enfermería de un hospital.

\section{METODOLOGÍA}

Para operacional izar el mejoramiento continuo del Departamento de Enfermería considerado como una organización compleja haremos girar el P.D.C.A. (que son las iniciales en inglés de planear, hacer, verificar y actuar) (CORRÊA, 1993, 
p.15). Simultáneamente se aplican los siete pasos del proceso de cambio propuesto por Lippitt (LEDDY \& PEPPER, 1985, p. 316), y que concuerdan de la siguiente manera:

- Planear:

1) Diagnóstico del problema.

2) Valoración de la motivación y de la capacidad de cambio

3) Valoración de la motivación y recursos del agente de cambio.

4) Selección de objetivos progresivos del cambio.

- Hacer: 5) Elección de la función apropiada del agente de cambio.

- Verificar: 6) Mantenimiento del cambio una vez iniciado.

- Actuar: 7) Terminación de la relación de ayuda.

\section{PROPUESTA TEÓRICA DE CAMBIO PARA EL MEJORAMIENTO CONTINUO DEL DEPARTAMENTO DE ENFERMERÍA COMO ORGANIZACIÓN COMPLEJA}

Para hacer girar el P.C.D.A. en concordancia con la teoría de cambio, partiremos de los pasos de la teoría de LIPPITT.

- PLANEAR implica:

1) Diagnóstico ya presentado en el análisis realizado.

2) Valoración de la motivación y de la capacidad de cambio suele presentarse cierta resistencial al cambio. Por lo que se deben revisar los factores culturales, sociales, relativos a organización, psicológicas que motivan la resistencia y trabajar la resistencia con estrategias como: confrontación que cuestiona las verdades sostenidas y reducir al mínimo la ansiedad con confianza y distracción. Identificar la disposición a aceptar el cambio de la persona según categoría: innovadores, adoptan pronto (miembros respetados); primeros adoptadores mayoritarios, los excépticos y los rezagados y los que lo repudian. Se debe alentar al personal de enfermería para que se ocupe de resolver su problema.

3) Valoración de la motivación y recursos del agente de cambio se debe tener los conocimientos y habilidades adecuados para motivar el cambio: educación permanente. Los líderes debense apoyar, con conciencia de cambio, educando siempre al personal. Sosteniendo una relación basada en la confianza y el respeto; trabajo en equipo. Es necesario contar con recursos financieros, físicos, materiales.

4) Selección de objetivos progresivos de cambio.

En la estructura:

Tipo: Fortalecer la prevención terciaria con programas educativos y organización de autogestión por asociación de pacientes. 
Subsistema: Establecer accesibilidad al manejo de la tecnología avanzada al equipo de enfermería y a los usuarios con sesiones terapéuticas para manejo de stress.

Fronteras Externas: Establecer líneas de investigación con capacitación de equipo para obtener tiempo y financiamiento y prestigio.

Internas: Procurar un cambio del modelo asistencial propuesto por el equipo para mejorar los índices de calidad de la atención.

Filosofía: Reorganizar la asistencia por tecnología intelectual y manual. La enfermera aplica teoría de cuidado según modelo asistencial establecido, reduciendo número de pacientes por enfermero para obtención de calidad. Participación de la auxiliar en administración de medicamentos con conciencia. Creer en los empleados.

Estilo de vida: Reunir del personal auxiliar y a las niñeras para identificar sus conocimientos, habilidades, actitudes y proponer formas de trabajo que impliquen compromiso con la calidad de atención.

En el proceso:

Adaptación al entorno: Promover programa de incentivos al personal por su labor en asistencia, educación e investigación con horarios, ambiente de trabajo. Educación continua a los clientes para evitar reingresos con análisis de factores culturales, sociales, psicológicos, de la organización.

Integración: Establecer los índices de calidad de enfermería para promover el reciclaje de los clientes de una unidad a otra. Plan estratégico para promover agilización de otros departamentos.

Toma decisiones: Educación continua para lideranza a todos los niveles. Ensayos de modelos de asistencia alternativos (plan piloto de enfermería).

Tratamiento de la información: Boletín de enfermería sobre auditorias del cuidado efectuado (registros archivados en computadora). Relatorios de experiencias en la asistencia (sesiones por unidad y por departamento).

Comunicación: Sesiones por unidad con enfermera de salud mental y personal sobre factores de stress y cómo enfrentarlo. Preparación al líder de cada unidad para post clínica con el personal (diaria) y revisar logros de calidad.

Codición-formación: Cursos de refrescamiento para ensayar modelos de asistencia (planeamiento - lideranza - evaluación de calidad - pesquisa ética publicaciones, conferencias, posters).

Compromiso: Reconocimiento escrito por labor realizada a los mejores y revisión de la calidad de asistencia de unidad por períodos rotatorios.

- HACER). Elección de un papel adecuado de agente de cambio: En esta etapa se ejecuta el cambio, se pone en juego la capacidad del líder para lograr el trabajo de equipo. Con miras a la calidad de atención. Es importante la comunicación y las estrategias de cambio. Hay que valorar la categoría de la estrategia de cambio que se va aplicar: empírica racional, normativa y reeducativa, de poder y coercitiva, propiciatoria, persuasiva o de poder. Se debe disponer de planes de contingencia para utilizarlos en caso de que el plan original presente problemas inesperados. 
- VERIFICAR). Mantenimiento del cambio: es la fase delicada del proceso. Se requiere tiempo para sentirse cómodo con el nuevo proceso y es posible que algunos deseen volver a las formas antiguas. Se deble respaldar el cambio y fortalecer el compromiso para continuar con este. Si parece necesario efectuar un gran cambio suele ser conveniente dividirlo en partes más pequeñas para ponerlo en práctica poco a poco.

- ACTUAR). Terminación de la relación de ayuda. Es conveniente evaluar conjuntamente el proceso de cambio y los resultados antes de dar por terminada la relación. Es necesario contar con nuevas expectativas de acuerdo a los cambios culturales, sociales, económicos, políticos del país y de la salud por reiniciar el proceso de manejo de las variaciones que afecten la calidad de atención.

\section{CONSIDERACIONES FINALES}

La asistencia de enfermería pediátrica tiente el reto de lograr calidad de atención en la que se perciba satisfacción del cliente y del enfermero.

La administración de la asistencia está influenciada por diferentes escuelas del pensamiento administrativo pero sin una clara visión del camino para lograr la calidad total. Al analizar la asistencia de enfermería como un sistema de organización compleja se pueden encontrar puntos fuertes y débiles en estructura y proceso.

Es importante indicar un camino factible para lograr calidad total en la asistencia de enfermería. Bien sea con el modelo de cambio de Levin o de Lippitt o con los principios de calidad total como base filosófica.

La aplicación de los mecanismos o estrategias de cambio es la etapa que falta para comprobar si el camino es el correcto.

\section{VIABILITY OF A PROPOSAL OF CHANGING AND IMPROVING A COMPLEX ORGANIZATION NURSING DEPARTMENT}

The nursing department is a complex organization, or a social system, made up of two or more groups interconnected through a complex net of social relations, common values and goals, used as the context for nursing interventions. The complex organization embodies type, subsystems, limitations, philosophy, life styles and process of adaptation to the environment, integration of subsystems, decision making, information processing, 
communications, coalition-formation and commitment. Theoretical interchange proposal to achieve full quality parameters is based on the seven stages of Lippit" exchange theory.

UNITERMS: system, complex organization, structure, process, interchange, full quality

\section{BIBLIOGRAFIA}

01. CORRÊA, P.A. Qualidade total, da visão à sistematização. Rio de Janeiro: Imagem.

02. HALL, J.; REDDING, B. Enfermería en salud comunitaria: un enfoque de sistemas. Washington: OPS, 1990.

03. LEDDY, S.; MAE P. Bases conceptuales de la enfermería profesional. Filadelfia: JB Lippincott, 1984.

04. WERNECK, D. O diagnóstico organizacional como elemento decisivo para a qualidade. Hospital. Administração e Saúde, v. 118, n. 2, março/abril. 1994. 